\title{
Characterization of a polymer gel dosimetry system based on n-isopropylacrylamide and n-n' methylenebisacrylamide
}

\section{F. Mattea ${ }^{1}$}

Instituto de Investigaciones de Córdoba, CONICET-UNC.

Facultad de Ciencias Químicas - Universidad Nacional de Córdoba Departamento de Química Orgánica.

E-mail: fmattea@gmail.com

\section{Strumia}

Instituto de Investigaciones de Córdoba, CONICET-UNC.

Facultad de Ciencias Químicas - Universidad Nacional de Córdoba Instituto Multidisciplinario de Biología Vegetal, IMBIV,

E-mail: mcsefcq. unc.edu.ar

\section{Valente}

Institute of Physics E. Gaviola - CONICET, Córdoba; Argentina

LIIFAMIRX - Laboratorio de Investigación e Instrumentación en Física Aplicada a la Medicina e Imágenes por Rayos X - University of Córdoba; Argentina

E-mail: valente@famaf.unc.edu.ar

Diagnostic radiology and radiotherapy are the most used techniques to detect and treat several pathologies like tumor and cancer diseases. The process of radiotherapy is complex and involves an understanding of medical physics, radiobiology, radiation safety, dosimetry, radiotherapy planning, simulation and interaction of radiation therapy with other treatment modalities. One of the key aspects of radiotherapy is to determine the real dose an organ or tissue is exposed. It is possible to measure this dose directly with the use of suitable phantoms and dosimeters. Among the different types of dosimeters, polymeric ones are of great interest because of their capability to mimic soft-tissue while maintaining a specific shape presenting high dose sensitivity and the ability to retain $3 \mathrm{D}$ spatial dose distribution for long periods of time after being exposed. In this work the preparation and characterization of a polymeric dosimeter based on the monomers $\mathrm{N}$ isopropylacrylamide (NIPAM), N-N'-methylenebisacrylamide (BIS) and an aqueous gelatin matrix containing Tetrakis(hydroxymethyl)phosphonium chloride (THPC) as oxygen scavenger is studied. Different characterization techniques, such as X-ray Raman spectroscopy, confocal and scanning electronic microscopy, and absorbance/transmitance analysis of the different dosimeters after being irradiated are presented and analyzed from a physical and chemical point of view, in the interest of proposing new materials for specific treatment purposes.

X Latin American Symposium on Nuclear Physics and Applications (X LASNPA)

December 1-6 2013

Montevideo, Uruguay

\footnotetext{
${ }^{1}$ Speaker
} 


\section{Introduction}

Diagnostic radiology and radiotherapy are the most used techniques to detect and treat several pathologies like tumor and cancer diseases. According to available evidence [1] at least $52 \%$ of cancer patients should receive radiotherapy during their treatment. The process of radiotherapy is complex and involves understanding of the principles of medical physics, radiobiology, radiation safety, dosimetry, radiotherapy planning and simulation. One of the key aspects of radiotherapy is to determine the real dose an organ or tissue is exposed. If the irradiation conditions are well defined and known in relation to the anatomy of the patient, dosimetry calculation is straightforward by means of treatment planning algorithms or Monte Carlo simulations. However, if those conditions are not fulfilled, it is also possible to measure the organ dose directly with the use of suitable phantoms and dosimeters [2]. Among the different types of dosimeters, polymeric ones are of great interest because of their capability to mimic soft-tissue while maintaining a specific shape presenting high dose sensitivity and the ability to retain 3D spatial dose distribution for long periods of time after being exposed [3]. A typical polymer gel dosimeter is a complex aqueous composition of physical gel-forming agent, monomers, and oxygen scavengers. The concentration of each component affects the sensitivity and the response of the dosimeter to radiation. Therefore, an in-depth study on the preparation methodology is essential for the development of new dosimeters.

In this work research regarding to the preparation and characterization of a polymeric dosimeter based on the monomers N-isopropylacrylamide (NIPAM), N-N'methylenebisacrylamide (BIS) and an aqueous gelatin matrix containing Tetrakis(hydroxymethyl)phosphonium chloride (THPC) as oxygen scavenger has been done. Different characterization techniques, such as X-ray Raman spectroscopy, confocal and scanning electronic microscopy, and absorbance/transmitance analysis of the different dosimeters after being irradiated are presented and analyzed from a physical and chemical point of view, in the interest of proposing new materials for specific treatment purposes.

\section{Materials and Methods}

Dosimeters were prepared with NIPAM 97\% and BIS 99\% provided by Sigma Aldrich® in an gel matrix constituted by porcine skin gelatin 300 Bloom produced by Fluka and an aqueous solution of tetrakis (hydroxymethyl) phosphonium chloride (THPC). A basic scheme of the dosimetry system studied in this work is depicted in Figure 1. 


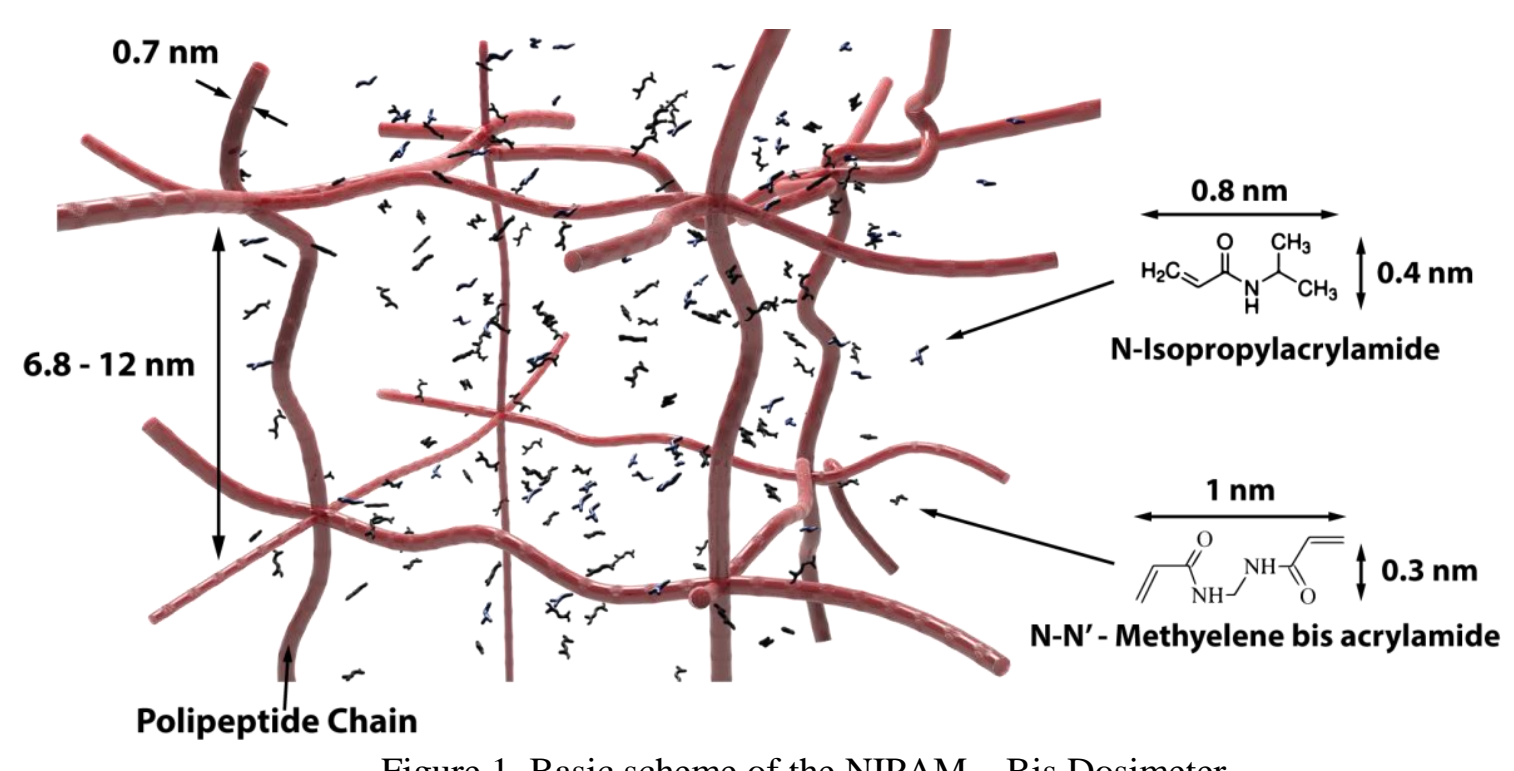

Figure 1. Basic scheme of the NIPAM - Bis Dosimeter

\subsection{Dosimeter preparation}

For the dosimeter preparation the quantities presented in Table 1 were used, first the Gelatin 300 Bloom was dissolved at ambient temperature and heated up to $50^{\circ} \mathrm{C}$ for 30 minutes, then the temperature was lowered to $37^{\circ} \mathrm{C}$ to avoid the polymerization of the monomers during the dosimeter preparation. BIS was mixed for 15 minutes and then the NIPAM was incorporated and stirred for another 15 minutes. Finally, $10 \%$ of the water volume with THPC also at $37^{\circ} \mathrm{C}$ was incorporated and the mixture was stirred for 30 minutes. The final solution was used to fill cuvette type vessels leaving no air at the top to avoid oxygen inhibition during the irradiation induced polymerization. Finally the dosimetry vessels were stored for $24 \mathrm{hr}$ at $4^{\circ} \mathrm{C}$ until the irradiation experiments for stabilization purposes.

Table 1. Typical dosimeters composition used in this work

\section{COMPONENT}

\begin{tabular}{|c|c|}
\hline H2O & 86.9 \\
\hline GELATIN & 5.0 \\
NIPAM & 5.0 \\
BIS & 3.0 \\
THPC & 0.1 \\
\hline
\end{tabular}

CHEMICAL FUNCTION

\section{Medium}

Gel Support

Monomer

Crosslinking agent

Oxygen Scavenger

\subsection{Dosimeter Irradiation}

Samples irradiations were carried out in a conventional X-ray tube with a $\mathrm{W}$ anode available at LIIFAMIR ${ }^{\otimes}$ facilities, which is thoroughly described elsewhere [4] and shown in 
Figure 2. The tube is connected to a generator with a maximum power of $3 \mathrm{~kW}$ that provides electrical current values from 5 to $60 \mathrm{~mA}$, and voltage values from 20 to $60 \mathrm{kV}$. Different dose levels can be achieved with this setup. In most of the irradiation experiments carried out a maximum electrical current of $40 \mathrm{~mA}$ and maximum voltage of $45 \mathrm{kV}$ were obtaining two different dose rates of $1.13 \mathrm{~Gy} / \mathrm{min}$ and $1.42 \mathrm{~Gy} / \mathrm{min}$. To measure the absolute dose rate a Farmer type ionization chamber (PTW-Freiburg TN 30013) was used, in water phantoms. Also, two different irradiation setups were used, first the samples were perpendicularly irradiated at each side of the cuvette for equal periods of time to achieve a more homogeneous dose distribution and the desired total irradiation time. Then, in-depth studies were attained irradiating only one side of the cuvette. Finally, specific irradiations of the cuvette dosimeters were performed implementing a dynamic modality using narrow collimation with the aim of testing the spatial dose distribution characteristics of the NIPAM-BIS system.
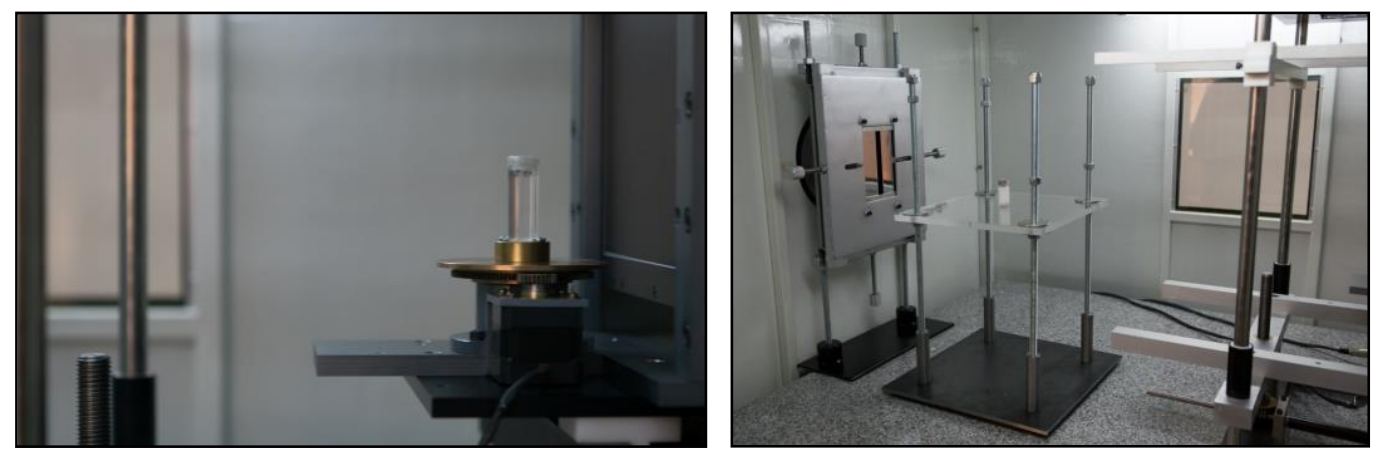

Figure 2. Irradiation setup for the cuvette type dosimeters.

\subsection{Dosimeter Characterization}

Once irradiated the dosimeters were characterized using a Raman spectrometer Labram HR (Horiba Jobin-Yvan), spectra were excited by $632.8 \mathrm{He}-\mathrm{Ne}$ laser with a $6.3 \mathrm{mV}$ power. Also a spectrophotometer S1205 (UNICO) apparatus was used to measure the absorbance of the irradiated material and a visible light optical transmission in-home apparatus [5] was used to characterize de polymerization degree in the irradiated dosimeters.

\section{Results and Discussion}

After the dosiemeters irradiation, macroscopic differences were cleary observed, as reported by figures $3 \mathrm{a}, 3 \mathrm{~b}$ and 4 . From Figure $3 \mathrm{a}$, it is possible to appreciate the macroscopic differences of the dosimeters irradiated at different doses from 0 Gy up to $56 \mathrm{~Gy}$, at plain sight the results show that for doses higher than 34 Gy the NIPAM-BIS dosimetry system used in this work becomes saturated. Figure $3 \mathrm{~b}$ shows the in-depth beam penetration of these dosimeters, in this case only one face of the dosimetry cells 
were irradiated at different doses at a dose rate of $1.42 \mathrm{~Gy} / \mathrm{min}$. Finally in Figure 4 the ability to retain the $3 \mathrm{D}$ dose distribution was tested, for this purpose the dosimeters were rotated with a $6 \mathrm{rpm}$ speed while irradiated and the difference from a non-irradiated sample, one where the X-ray beam was collimated and a fully irradiated sample is presented.
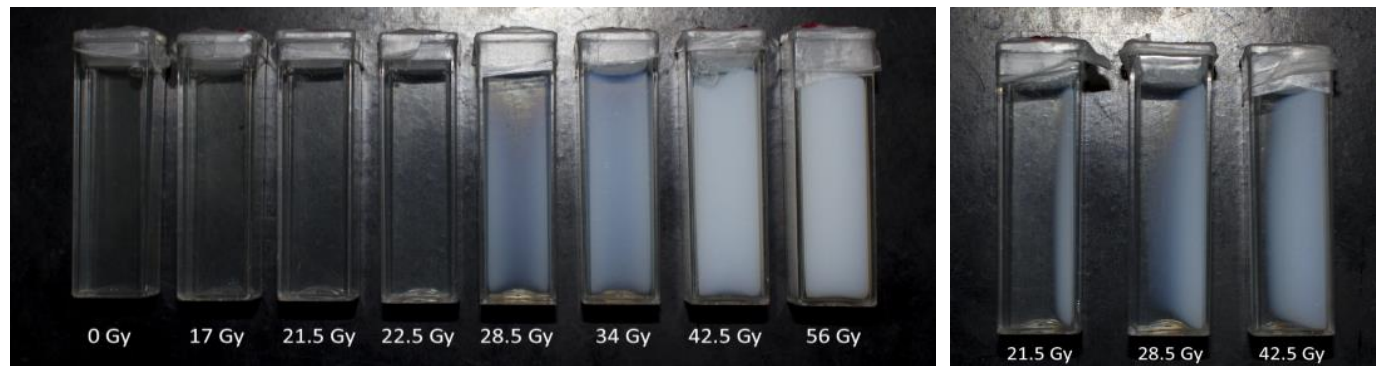

Figure 3a (left). NIPAM-BIS based dosimeters treated at different doses from 0 Gy to $56 \mathrm{~Gy}, 3 \mathrm{~b}$ (right) In-depth penetration results

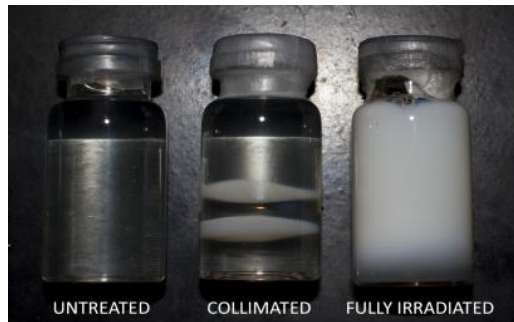

Figure 4. 3D dose dsitribution results

Raman spectroscopy of the irradiated dosimeters was performed in order to determine the disappearance of NIPAM and BIS monomers and consequently the polymerization within the dosimetric system. Figure 5 shows a typical spectrograph of the NIPAM-BIS dosimeter and the characteristic peaks of the most important functional groups present in this system.

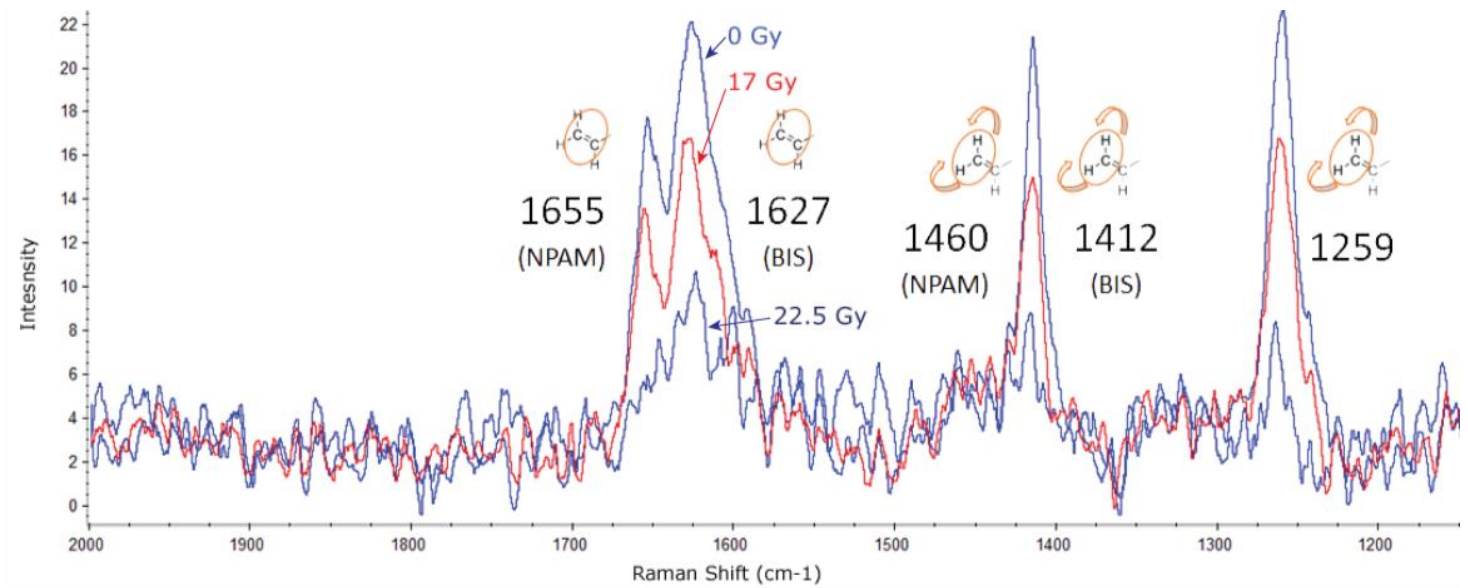

Figure 5. Raman spectrograph of NIPAM-BIS based dosimeters. 
From the Raman analysis of the dosimeters presented in Figure 3a, the area of the representative peaks of the vinyl groups in the NIPAM and BIS monomers were quantified, relative to the area of a non-reactive group in the molecules for normalization purposes. In Figure 6a these areas at the different doses assayed in this work are represented. The vinyl groups are the molecular places where the radical polymerization takes place and as a result from this radiation induced reaction a BIS monomer can be connected to the NIPAM molecules and form a 3D gel-type structure. During the irradiation the presence of these vinyl groups becomes less noticeable as more reaction takes place. The disappearance degree of these functional groups considering the initial concentration of the monomers and the fact that each BIS molecules has 2 vinyl groups while NIPAM molecules have only one, is depicted in Figure 6b. The trend observed in these figures shows that for doses higher than $34 \mathrm{~Gy}$ the analysis is not reliable and the dosimeters have been used above their saturation range as it was indicated from the visual overview of the dosimeters.
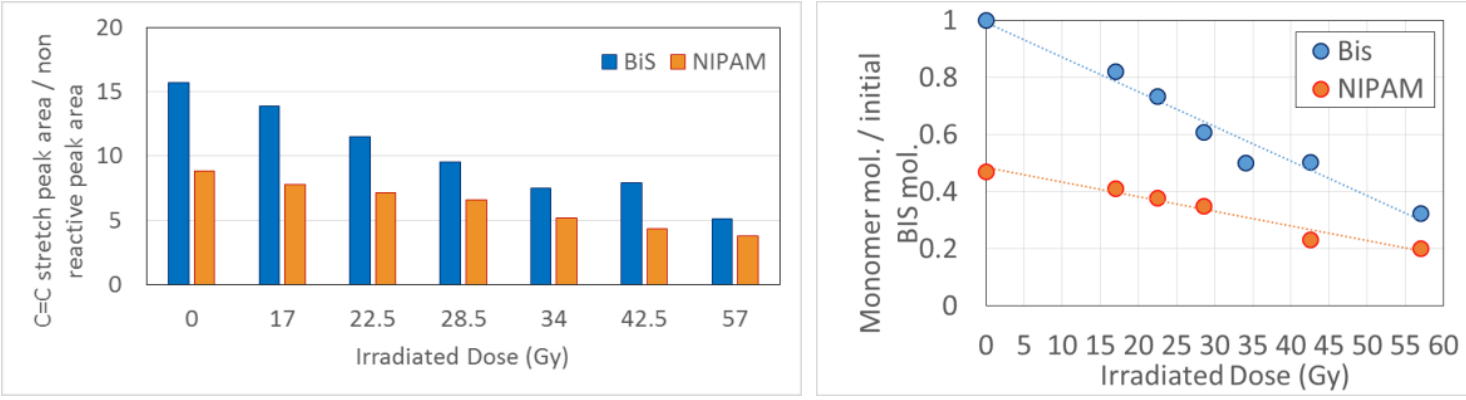

Figure 6a. (left) Bis and NIPAM representative peak areas for irradiated dosimeters. $6 \mathrm{~b}$ (right) Monomers disappearance degree.

Figure 7 reports the obtained results for the optical density difference of the irradiated dosimeters at two different dose rates. The results suggest a linear trend for both cases in a similar way that the chemical interpretation from the Raman spectroscopy results

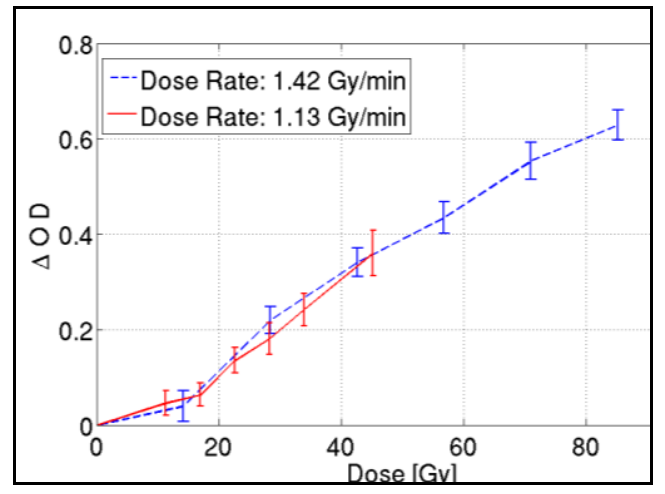

Figure 7. Optical difference density results. 


\section{Conclusions}

NIPAM - BIS based systems for X-ray dosimetry applications were succesfully elaborated. Different characterization techniques were applied to the irradiated dosimeters obtaining similar results from a chemical and optical point of view. The dose response characterization indicated that the studied dosimetric system can be used within a dose range of 0 to 34 Gy with great reliability. In view of the obtained results it was demonstrated the capability to accomplish the synthesis, elaboration, irradiation and characterization of polymer gel systems.

\section{Acknowledgements}

This study was partially financed by the Argentinean Government by means of the PIP 112-20110101029 and PIP 11420090100398 projects, and the PICT 2011-0654 FONCyT Res 140/12 and PICT 2008/0398 ANPCyT projects. The authors would also like to thank PME (2006) 1544 grants for Raman measurements in LANN of SNM.

\section{References}

[1] G. Delaney, S. Jacob, C. Featherstone, M. Barton, The role of radiotherapy in cancer treatment, Cancer, 104 (2005) 1129-37.

[2] A. Meghzifene, D.R. Dance, D. McLean, H.-M. Kramer, Dosimetry in diagnostic radiology, European Journal of Radiology, 76 (2010) 11-14.

[3] C. Baldock, Y.D. Deene, S. Doran, G. Ibbott, A. Jirasek, M. Lepage, K.B. McAuley, M. Oldham, L.J. Schreiner, Polymer gel dosimetry, Physics in Medicine and Biology, 55 (2010) R1.

[4] J. Vedelago, M. Valente. Modelo y desarrollo instrumental para dosimetria de Fricke gel in situ para ambito clinico, Proceedings of 98th Argentine Physics Association Meeting (2013) p. 31.

[5] J. Vedelago. Modelo teórico y desarrollo instrumental para dosimetría Fricke gel in situ de alta performance para ámbito clínico. Graduation thesis, Universidad de Córdoba, Argentina, 2013. 